\title{
Attention distribution as a function of novelty and familiarity'
}

\section{MICHAEL LEWIS, SUSAN GOLDBERG AND MARILYN RAUSCH}

FELS RESEARCH INSTITUTE

Experimentally defined novelty and familiarity was shown to influence the distribution of attention to visual stimuli in the 42-month-old child. Familiarity produced response decrement while novelty resulted in response recovery. Further, attentive behavior was maintained for approximately $30 \mathrm{~min}$. by varying the environmental input.

The dimension of novelty and familiarity is an important stimulus property influencing attentional behavior (Berlyne, 1960; Sokolov, 1963; Cantor, 1965). One definition of novelty and familiarity is based on the assumed frequency with which certain stimuli have occurred in the organism's experiential history (Lewis, 1965). Experimentally, novelty and familiarity can be defined by controlling the frequencies with which stimuli occur. A stimulus $S_{1}$ is defined as familiar when it has been presented repeatedly for $n$ trials. The occurrence of a second stimulus, $S_{2}$, on trial $n+1$ defines $S_{2}$ as a novel event. $S_{2}$ can be any event discriminable from $s_{1}$. The present experiment, using visual stimuli, seeks to investigate this problem with pre-school children.

Method

Twenty Ss, 10 experimental and 10 controls, 3-1/2 years of age, were each seated at a table enclosed in a uniform gray room approximately $5 \times 5 \mathrm{ft}$. The visual stimuli were presented, by rear-screen projection, approximately 2-1/2 ft. from $S$ 's head. Total fixation time (TF) or the total time $S$ oriented his head and eyes toward the screen was recorded by two independent observers (interobserver reliability, $\mathrm{r}=.94$ ).

Four different sets of stimuli were presented (see Fig. 1) and the order of presentation was the same for both experimental and control groups. Set A was presented first, followed by sets $B, C$ and $D$. The control group received seven trials of $A_{1}$, each trial $30 \mathrm{sec}$. in duration with a $30-\mathrm{sec}$. intertrial interval. The experimental group received six trials of $A_{1}$, followed by one trial of $A_{2}$. This procedure was the same for each set.

\section{Results}

Response Decrement. Figure 2 presents the mean TF for each trial for each set. The response to familiarity for each set (see trials 1-6) is response decrement. A linear function, $y=a x+b$, with a relatively steep negative slope fits the data; $r^{2}$ varies from .61-.81 and .61-.69 for the experimental and control groups respectively. Between-set differences were determined by obtaining trial 1-6 differences for each $S$ for each set and comparing this distribution by a Friedman two-way analysis of variance (Siegel, 1956). No significant set differences in response decrement for either the control or experimental groups was found. Moreover, no experimentalcontrol group differences in response decrement were found for any of the sets.

Response Recovery. The response to a novel stimulus can be determined by observing the difference between the predicted $T F$ on the regression line and the observed TF. The criteria of response recovery was an increase of more than two SD from the predicted point. The control group showed no significant increases whereas the experimental group's data was: $\mathrm{A}_{2}\left(4.7 \mathrm{SD}, \mathrm{p}<.5 \times 10^{-6}\right) ; \mathrm{B}_{2}\left(2.8 \mathrm{SD}, \mathrm{p}<.5 \times 10^{-3}\right)$;
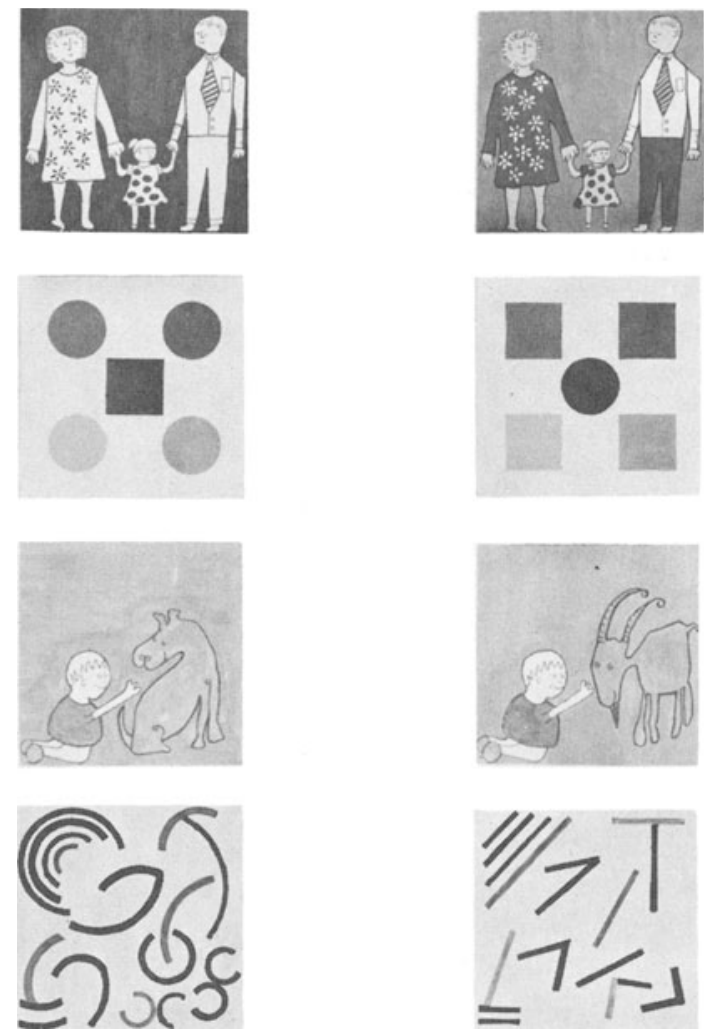

Fig. 1. Visual stimuli shown in sets from $A$ (top) to D (bottom). $s_{1}$ is shown on the left, $S_{2}$ on the right for each set. All stimuli except $S_{1}$ in set $A$ are chromatic. For set $A$ the violation was the change from an achromatic to a chromatic picture; for set $B$, the violation was a change in form; for set $C$, content; and for set $D$, curvature. 


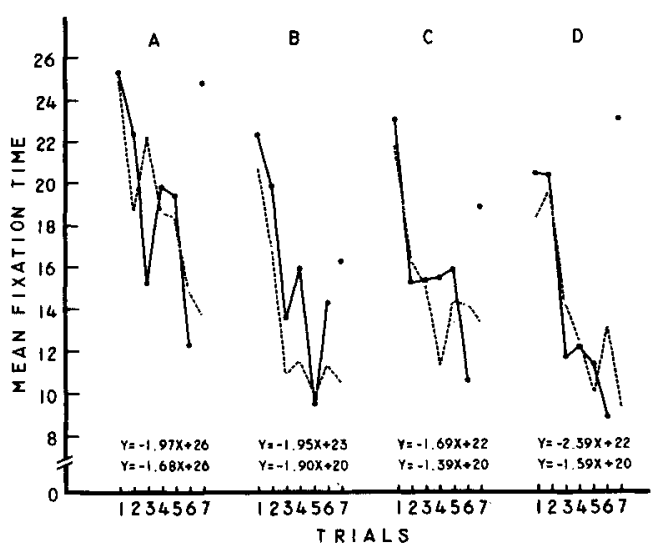

Fig. 2. Mean total fixation time for each trial, stimulus sets A-D for experimental group (solid line) and control group (dotted line). The seventh trial is indicated by an isolated point for the experimental group. For each set, the lower equation represents a linear function for the control group, the upper equation, the experimental group.

$\mathrm{C}_{2}\left(4.0 \mathrm{SD}, \mathrm{p}<.5 \times 10^{-5}\right) ;$ and $\mathrm{D}_{2}(8.6 \mathrm{SD}, \mathrm{p}<.5 \mathrm{x}$ $10^{-10}$ ). For both experimental and control groups response recovery also occurred whenever sets changed. This is seen most clearly for the control group for trial 1 of each new set which shows a significant increase for each change (by sign test, $\mathrm{p}<.01$ )

\section{Discussion}

TF for the 3-1/2-year-old can be considered part of the orienting reflex in that response decrement occurs to repeated stimulation $\left(S_{1}\right)$ and recovery occurs when $S_{1}$ is varied $\left(S_{2}\right)$. Sokolov $(1963)$ argues that response decrement and recovery are mediated by some central process such as memory or neuronal model formation. Novelty is defined as the lack of match between the external event and the model and results in attentive behavior. Within this theoretical system, rate of response decrement can be viewed as a function of the speed of model acquisition whereas response recovery is a function of discrimination ability, model formation and the nature and degree of the violation $\left(\mathrm{S}_{2}\right.$-distortion, change in complexity, form, shape, color, etc.). The present study shows that distribution of attention can be a function of novelty and familiarity. Further, attention can be sustained over relatively long periods (in this case, for $30 \mathrm{~min}$.) by varying the visual input.

\section{References}

Berlyne, D. E. Conflict, arousal and curiosity. New York: McGrawHill, 1960.

Sokolov, Ye. N. Perception and the conditioned reflex. Trans, by S. W. Waydenfeld. New York: MacMillan Co., 1963.

Cantor, G. N. Responses of infants and children to complex and novel stimulation. In Lewis P. Lipsitt and Charles C. Spiker (Eds.), Advances in child development and behavior. New York: Academic Press, Vol. 1, 1963.

Lewis, M. Exploratory studies in the development of a face schema. Paper presented at the Symposium on the Origins of Social Behavior, American Psychological Association Meeting, Chicago, 1965.

\section{Note}

1. This research was supported in part by Grants No. HD-00868, FR-00222 and FR-00537 from the National Institute of Mental Health, United States Public Health Service. The authors wish to thank Helen Campbell for her aid in collecting data. 